\title{
Using Ohm's Law to Calibrate a Picoammeter to 0.4 Pico-ampere Precision
}

\author{
Joseph Hashem, ${ }^{\text {a) }}$ Tiankuan Liu,${ }^{\text {b) }}$ Zhihua Liang, ${ }^{\mathrm{c}}$ and Jingbo $\mathrm{Ye}^{\mathrm{d})}$ \\ Department of Physics \\ Southern Methodist University \\ Dallas, Texas 75275 USA
}

Received: April 20, $2010 \quad$ Accepted: September 14, 2010

ABSTRACT

We present an experimental method based on Ohm's Law to calibrate the Keithley 6485 picoammeter with the calibration error less than 0.4 pico-ampere $(\mathrm{pA})$, which is specified as the highest sensitivity of this instrument. The maximum calibration difference between the measurement current and the calibration current in the picoammeter's 2 nano-ampere $(n A)$ range is $0.25 \mathrm{pA}$. This difference is far less than the highest accuracy, $0.4 \mathrm{pA}$, specified by the instrument. Measurement errors are estimated and discussed.

\section{INTRODUCTION}

Direct Current (DC) electrical current is measured with an ammeter. A precision digital multimeter with $61 / 2$ digits, like the Model 34401A from Agilent Technologies, can measure electrical current to an accuracy of 2 micro-ampere $(\mu \mathrm{A})$ in one year range at a temperature of $23 \pm 5^{\circ} \mathrm{C}$. These time and temperature conditions apply to the other instruments throughout this paper [1]. The state-of-theart SourceMeter, like the Model 2410 series from Keithley Instruments, Inc., can measure DC current with an accuracy as low as 300 pico-ampere (pA) [2]. However, many applications require higher precision. For example, one commonly uses a picoammeter like the Model 6485 from Keithley Instruments, Inc. to measure the leakage current of a diode. The measurement accuracy in the $2 \mathrm{nA}$ range of this picoammeter can reach down to $0.4 \mathrm{pA}$ [3]. The measurement accuracies of these instruments are listed in Table I in Section II. The purpose of this article is to discuss the

a)Email: jhashem@smu.edu

b)Email: liu@physics.smu.edu

c)Email: liangzhihua@gmail.com

d) Email: yejb@physics.smu.edu nontrivial calibration of the Keithley 6485 picoammeter. In the calibration process we also develop a method to reliably use this instrument in sub-pA measurements.

\section{CALIBRATION PRINCIPLE}

We employ Ohm's Law, $R=V / I$, to calibrate the picoammeter. With a given resistor, $R$, and a given voltage, $V$, the current through the resistor is calculated to be $I_{\text {cal }}=V / R$. By measuring the voltage, $V$, through the SourceMeter and knowing, precisely, the resistance, $R$, we can then measure the current, $I_{6485}$, by the picoammeter and compare $I_{\text {cal }}$ with $I_{6485}$ in order to calibrate the picoammeter. However, the precision of the resistor given by the manufacturer is not precise enough. Note that in order to generate an ultra low current, the resistance must be very large. The ultra high resistance measurement is not trivial.

We assume that the resistance of the resistor remains constant throughout the calibration process. With this assumption, we should obtain a straight line in the $I-V$ plot when both the voltage and the current are measured correctly. The resistor we choose is a 100 Giga-ohm (G $\Omega$ ) resistor of $0.5 \mathrm{~W}$ and $2 \%$ precision [4]. The exact value of the resistor is measured during the 


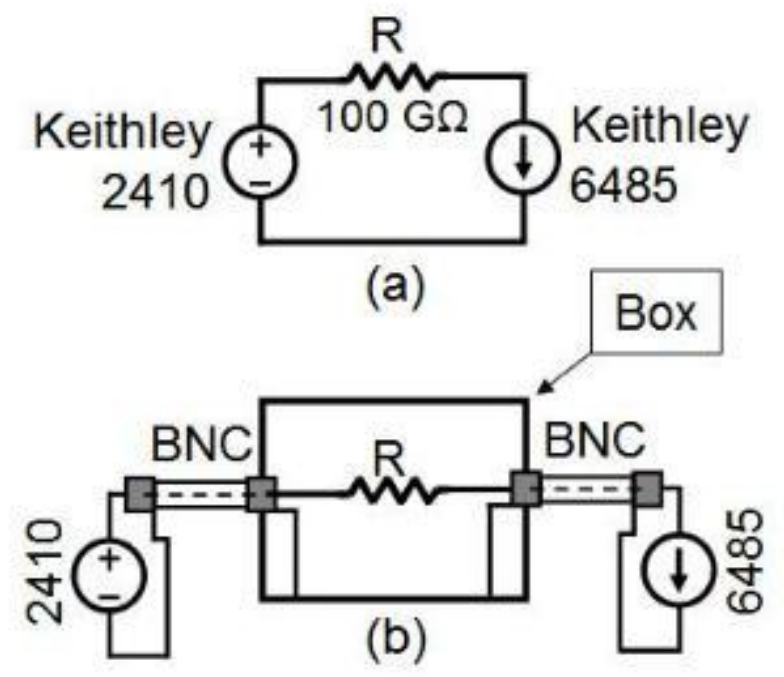

Figure 1. Schematic (a) and connection block diagram (b) of the picoammeter calibration.

calibration. The value of the resistance does not actually affect the measurement as long as one given resistor is used consistently. The highest power consumption in the resistor is approximately 10 micro-Watt with $1000 \mathrm{~V}$ voltage over it. This power consumption is very small and is considered to be dissipated quickly to the environment, and hence, does not contribute to any significant temperature increase of the resistor.

Our calibration of the picoammeter is done in two steps. The first step of the calibration measures the resistance. We determine the voltage by the SourceMeter and measure the current both by the SourceMeter as $I_{2410}$ and by the picoammeter as $I_{2 \mathrm{nA}}$ and $I_{20 \mathrm{nA}}$ in $2 \mathrm{nA}$ and 20
$\mathrm{nA}$ ranges respectively. For practical reasons, only the Keithley 6485 picoammeter's $2 \mathrm{nA}$ and $20 \mathrm{nA}$ current measurement ranges are used in the calibration. Ranges above $20 \mathrm{nA}$ are relatively easily calibrated and are not discussed in this paper. We then plot the resulting $I-V$ curves. We assume that $I_{2410}$ is correct above $300 \mathrm{pA}$, which is the highest instrument accuracy specified in the datasheet. $^{2}$ We verify that $I_{2 n A}$ and $I_{20 n A}$ above $300 \mathrm{pA}$ are consistent with $I_{2410}$ within the instrument accuracy of the Keithley Models 2410 and 6485 . The voltage that generates $300 \mathrm{pA}$ through the calibration resistor is $30 \mathrm{~V}$. We then use the voltage readings above $30 \mathrm{~V}$ and the current

\begin{tabular}{ccl}
\hline Model & Range & Accuracy \\
\hline $34401 \mathrm{~A}$ & $10 \mathrm{~mA}$ & $\pm(1 \times 0.05 \%+2 \mu \mathrm{A})$ \\
2410 & $1 \mathrm{uA}$ & $\pm(1 \times 0.029 \%+300 \mathrm{pA})$ \\
6485 & $2 \mathrm{nA}$ & $\pm(1 \times 0.4 \%+0.4 \mathrm{pA})$ \\
6485 & $20 \mathrm{nA}$ & $\pm(1 \times 0.4 \%+1 \mathrm{pA})$ \\
2410 & $200 \mathrm{mV}$ & $\pm(\mathrm{V} \times 0.012 \%+300 \mu \mathrm{V})$ \\
& & \\
\hline
\end{tabular}

Table I. Measurement accuracies of Agilent 34401A, Keithley 2410, and Keithley 6485. 


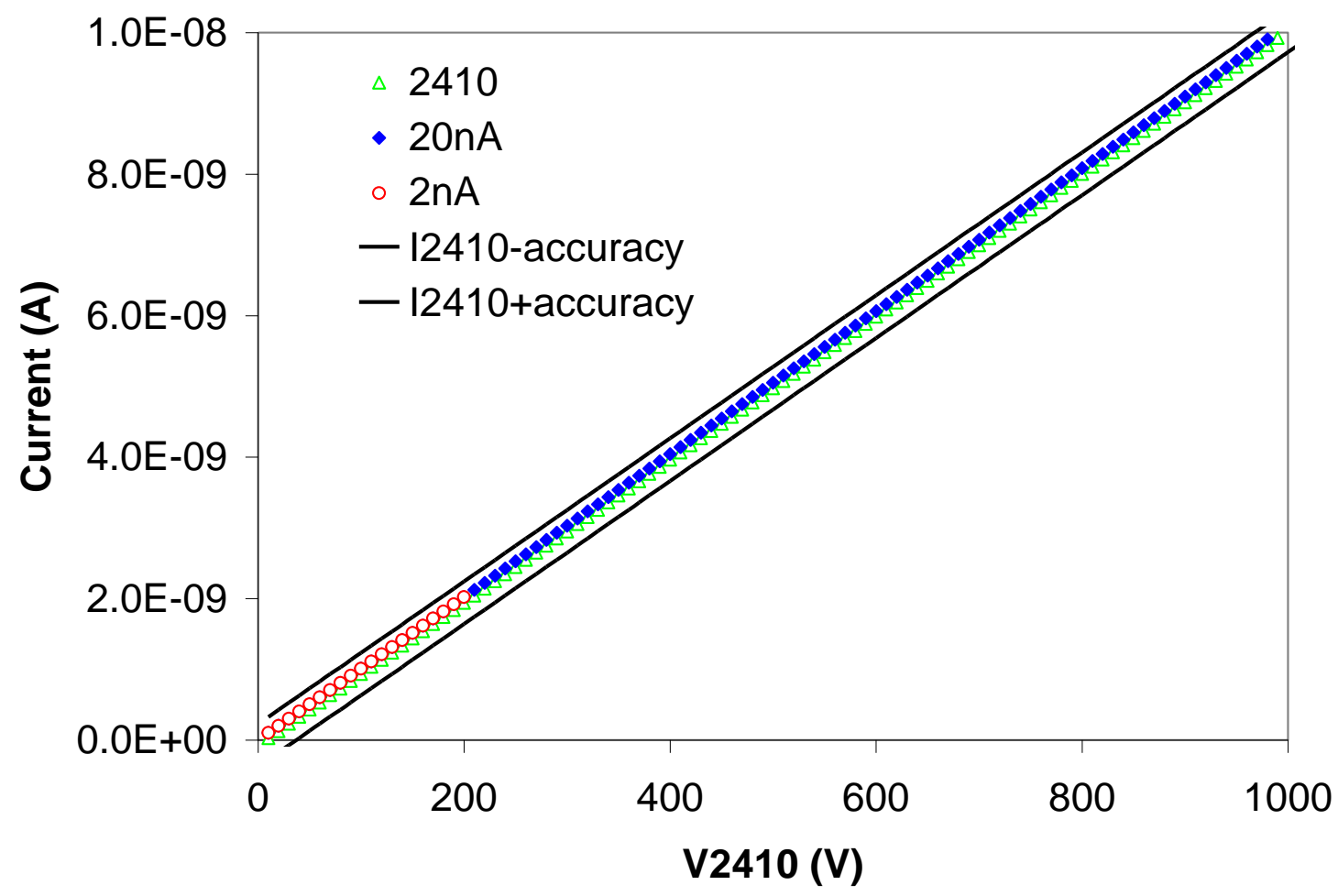

Figure 2. The current-voltage curves.

readings above $300 \mathrm{pA}$ to fit straight lines for each of the $I-V$ curves. The intersection of each straight line with the $I$ axis is taken to be the system error of each model. The slope of each fitted straight line is the conductance, and the reciprocal of this conductance is the resistance. We then use the weighted average of the three conductance measurements in order to calculate the resistance, $R$, and use it in the next calibration step.

The second step of the picoammeter calibration is to extrapolate each straight line of the $I-V$ curves below the threshold. We attribute any deviations from each straight line to the incorrect measurement of the current by the picoammeter. Hence, we are able to calibrate the picoammeter.

The voltage measurement accuracy of Model 2410 is listed in Table I. In order to generate $0.4 \mathrm{pA}$ through the $100 \mathrm{G} \Omega$ resistor, the voltage needs to be set at 40 $\mathrm{mV}$. According to the datasheet, the accuracy of this voltage is $304.8 \mu \mathrm{V}$. This translates to an uncertainty of 6.08 femtoampere $(f A)$ in the current calculation, or
$1.5 \%$ of $0.4 \mathrm{pA}$. We attribute the uncertainty to one of our calibration errors. Other errors will be discussed in Section IV, along with the calibration results.

\section{CALIBRATION SETUP}

A circuit schematic and a connection block diagram are shown in Fig. 1. We use Keithley's high voltage SourceMeter, Model 2410, with voltage and current read back, to provide the voltage up to $1000.00 \mathrm{~V}$. An ultra high resistance, high voltage, precision, and thick film resistor, RX-1M1009GE from Ohmite Manufacturing Company, is housed inside an aluminum project box. The picoammeter, Keithley 6485, and the high voltage Keithley 2410 SourceMeter, are connected through coaxial cables with BNC connectors. The aluminum project box and the coaxial cables provide shielding against electromagnetic noise throughout the entire system. This is crucial in current measurements within $\mathrm{pA}$ range or below.

Voltage from $2 \mathrm{mV}$ to $1000 \mathrm{~V}$ is set and measured by Keithley 2410 . The current 


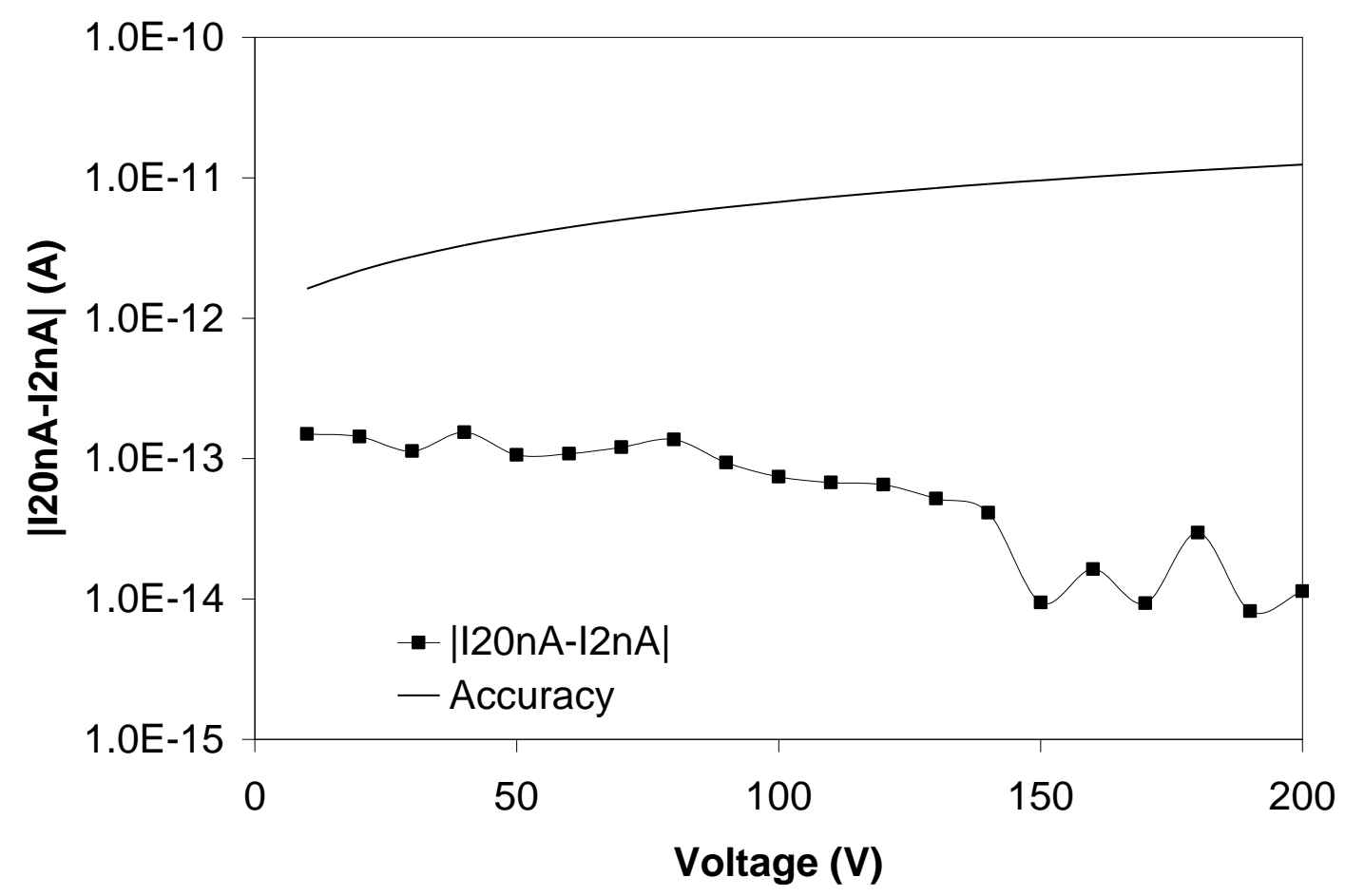

Figure 3. $\left(\left|I_{20 n A^{-}} I_{2 n A}\right|\right)$ and $\sqrt{\left(\Delta I_{20 n A}\right)^{2}+\left(\Delta I_{2 n A}\right)^{2}}$.

is measured by Keithley 2410 in $1 \mu \mathrm{A}$ range and Keithley 6485 in $2 \mathrm{nA}$ and $20 \mathrm{nA}$ ranges. Below $200 \mathrm{~V}$, the current is measured by Keithley 2410 and Keithley 6485 in 2 nA and 20 nA ranges. Above 200 $\mathrm{V}$, the current is measured only by Keithley 6485 in 20 nA range and Keithley 2410.

In order to acquire data from the picoammeter and the SourceMeter, a National Instruments LabVIEW program was constructed. The LabVIEW program automatically incremented the supply voltage, and read out the voltage and currents from both the SourceMeter and the picoammeter. At each set voltage, the voltage and current were measured 100 times. The averaged current and the standard deviation for each set of 100 current measurements are calculated in the same LabVIEW program. The standard deviation is used as the measurement error in the linear fitting.

\section{CALIBRATION RESULTS}

$I-V$ curves above $30 \mathrm{~V}$ are shown in Fig. 2. Each point shown in Fig. 2 is the average value. The lines in Fig. 2 are the current uncertainty range set by the SourceMeter, i.e., $I_{2410}+\Delta I_{2410}$ and $I_{2410}$ $\Delta I_{2410}$, where $\Delta I_{2410}$ is the measurement accuracy of the SourceMeter. As can be seen in Fig. 2, the current measurements of the picoammeter in $20 \mathrm{nA}$ and $2 \mathrm{nA}$ ranges all fall within the SourceMeter uncertainty range. This indicates that the picoammeter and the SourceMeter have consistent current measurement within their accuracy specification.

Shown in Fig. 3 is the current difference measured by the picoammeter in $2 \mathrm{nA}$ and $20 \mathrm{nA}$ ranges, $\left(\mid I_{20 \mathrm{nA}}-I_{2 \mathrm{nA}}\right)$, and the square root of the sum of the squares (RSS) of the accuracies in these two ranges, $\sqrt{\left(\Delta I_{20 n A}\right)^{2}+\left(\Delta I_{2 n A}\right)^{2}}$, where $\Delta I_{20 n A}$ and $\Delta I_{2 n A}$ are the accuracies of the picoammeter in these two ranges. The current difference is far less than the sum of the accuracies in these two ranges, indicating that the current measurements of the picoammeter in the two ranges are consistent with each other in their accuracy specification. As discussed in Section II, because we assume that the 


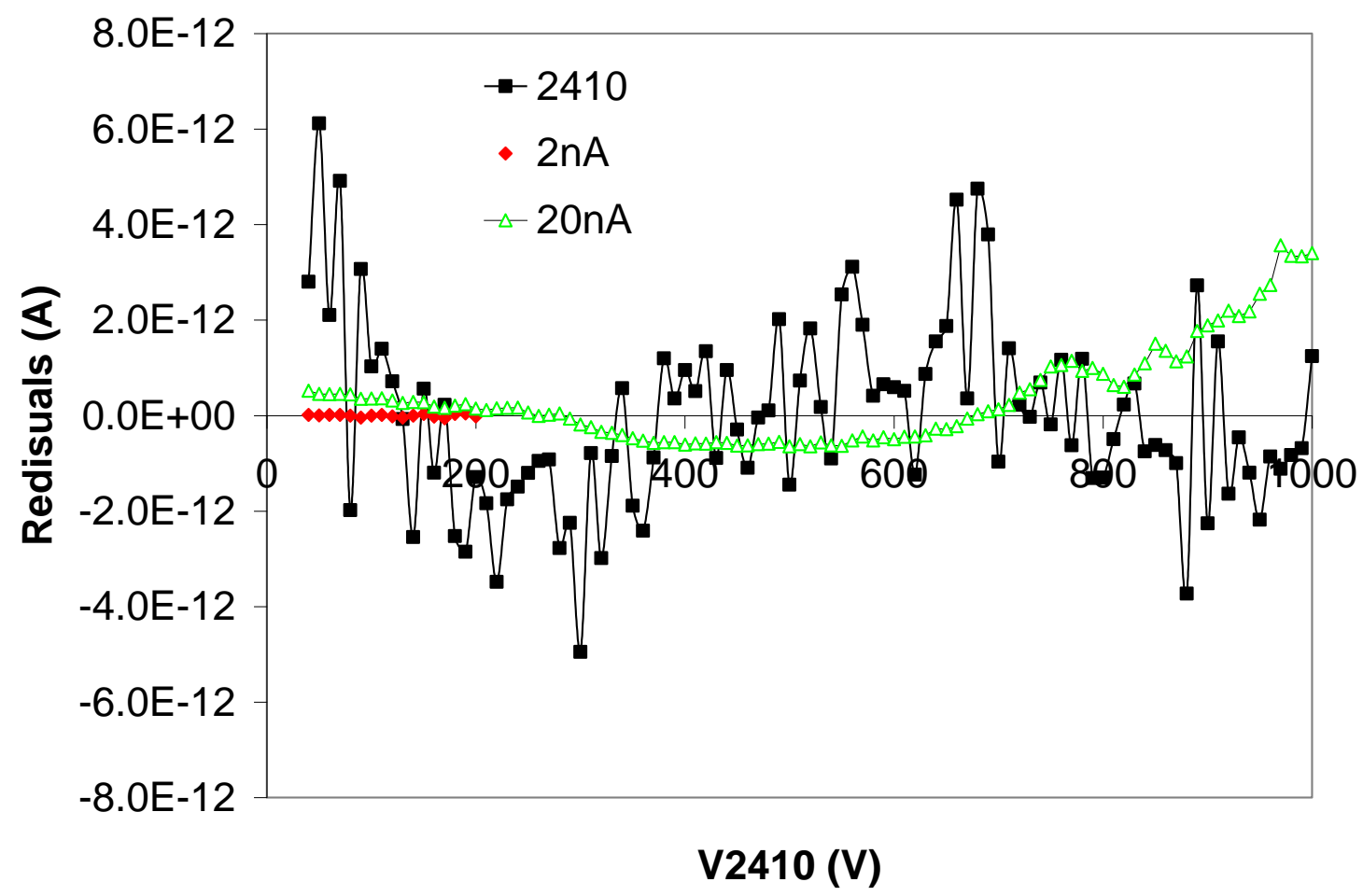

Figure 4. The fitting residuals of I-V curves.

SourceMeter measures the current correctly, we believe that the current measurements above $30 \mathrm{~V}$ in $2 \mathrm{nA}$ and $20 \mathrm{nA}$ ranges by the picoammeter are also correct.

Once we verified that the picoammeter current measurements over 30 $\mathrm{V}$ are correct in $2 \mathrm{nA}$ and $20 \mathrm{nA}$ ranges, we perform the curve fitting. The fitting process is to minimize the square of the sum of residuals, i.e., $\min \sum_{k=1}^{N} \frac{\left(I_{k}-Y V_{k}-I_{0}\right)^{2}}{\left(\Delta I_{k}\right)^{2}+Y^{2}\left(\Delta V_{k}\right)^{2}}$. Note that both the current and voltage values have measurement errors; therefore, this is not a normal least square linear curve fitting. Our goal, however, is to fit a straight line. The fitting is done by ROOT [5]. The fitting residuals, $\left(I-Y V-I_{0}\right)$, are shown in Fig. 4.

The fitting results are listed in Table II. The intersection $I_{0}$ is the system error. Comparing $I_{0}$ in Table $I$ and measurement accuracies in Table II, we conclude that system error, $I_{0}$, is within the accuracy specified for each instrument.
The slope of the fitting straight line is the conductance, which is the reciprocal of the resistance. The comparison of the fitted conductance values is shown in Fig. 5 . The distance between conductance values is within two times of the standard deviation. Since there is only one resistor, three conductance values are averaged based on their errors in order to calculate the average conductance $\mathrm{Y}$, as indicated in Eqs. (1) and (2):

$$
Y=\frac{\frac{Y_{2410}}{\left(\Delta Y_{2410}\right)^{2}}+\frac{Y_{2 n A}}{\left(\Delta Y_{2 n A}\right)^{2}}+\frac{Y_{20 n A}}{\left(\Delta Y_{20 n A}\right)^{2}}}{\frac{1}{\left(\Delta Y_{2410}\right)^{2}}+\frac{1}{\left(\Delta Y_{2 n A}\right)^{2}}+\frac{1}{\left(\Delta Y_{20 n A}\right)^{2}}},
$$

$$
\Delta Y=\frac{1}{\sqrt{\frac{1}{\left(\Delta Y_{2410}\right)^{2}}+\frac{1}{\left(\Delta Y_{2 n A}\right)^{2}}+\frac{1}{\left(\Delta Y_{20 n A}\right)^{2}}}}
$$




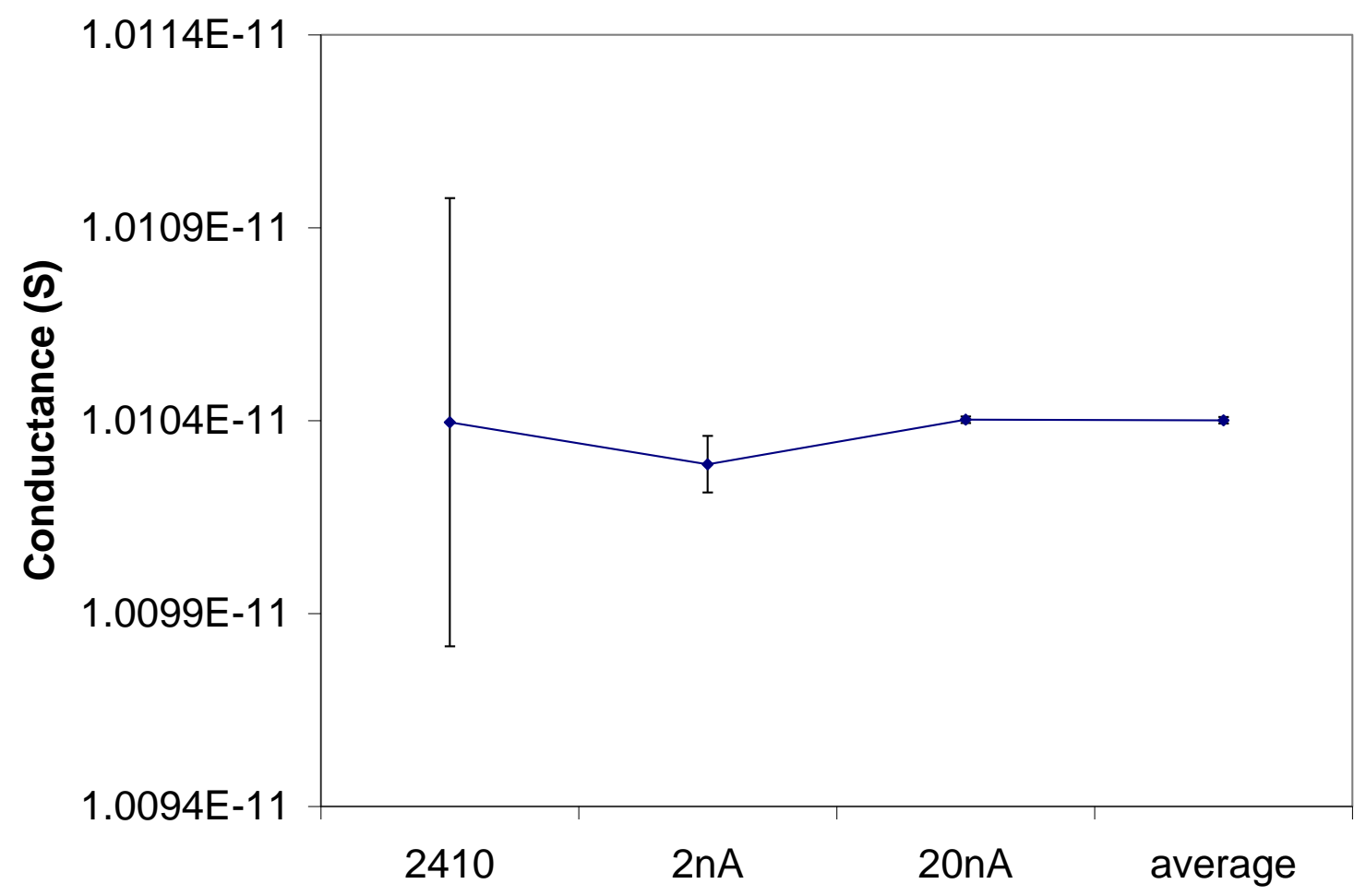

Figure 5. The comparison of the fitting conductance.

The resistance, $R$, is calculated by the equation $R=1 / Y=(989706.3 \pm 8.2) \times$ $10^{5} \Omega$. This is within the specification of the resistor.

Once we obtain the resistance, we extrapolated each straight line and calculated the calibration current $I_{c a l}=Y V+I_{0}$. The errors, $\mid I-I$ call, are shown in Fig. 6 and compared with the accuracy. As seen in Fig.
6 , the difference between the measurement current and the calibration current are within the accuracy specification, indicating that the current measurements in these two ranges are correct. The maximum calibration error is $0.25 \mathrm{pA}$ in $2 \mathrm{nA}$ range, far less than the highest accuracy, $0.4 \mathrm{pA}$, specified in Table I.

\begin{tabular}{ccc}
\hline & $Y(S)$ & $I_{0}(A)$ \\
\hline SourceMeter & $(10104.0 \pm 5.8) \times 10^{-15}$ & $-(78.5 \pm 3.1) \times 10^{-12}$ \\
PA 2 nA & $(101028.7 \pm 7.3) \times 10^{-16}$ & $-(5.9 \pm 8.8) \times 10^{-14}$ \\
PA 20 nA & $(1010402.1 \pm 8.4) \times 10^{-17}$ & $-(45.7 \pm 3.6) \times 10^{-14}$ \\
Average & $(1010400.8 \pm 8.3) \times 10^{-17}$ & N/A \\
\hline
\end{tabular}

Table II. Curve fitting results. 

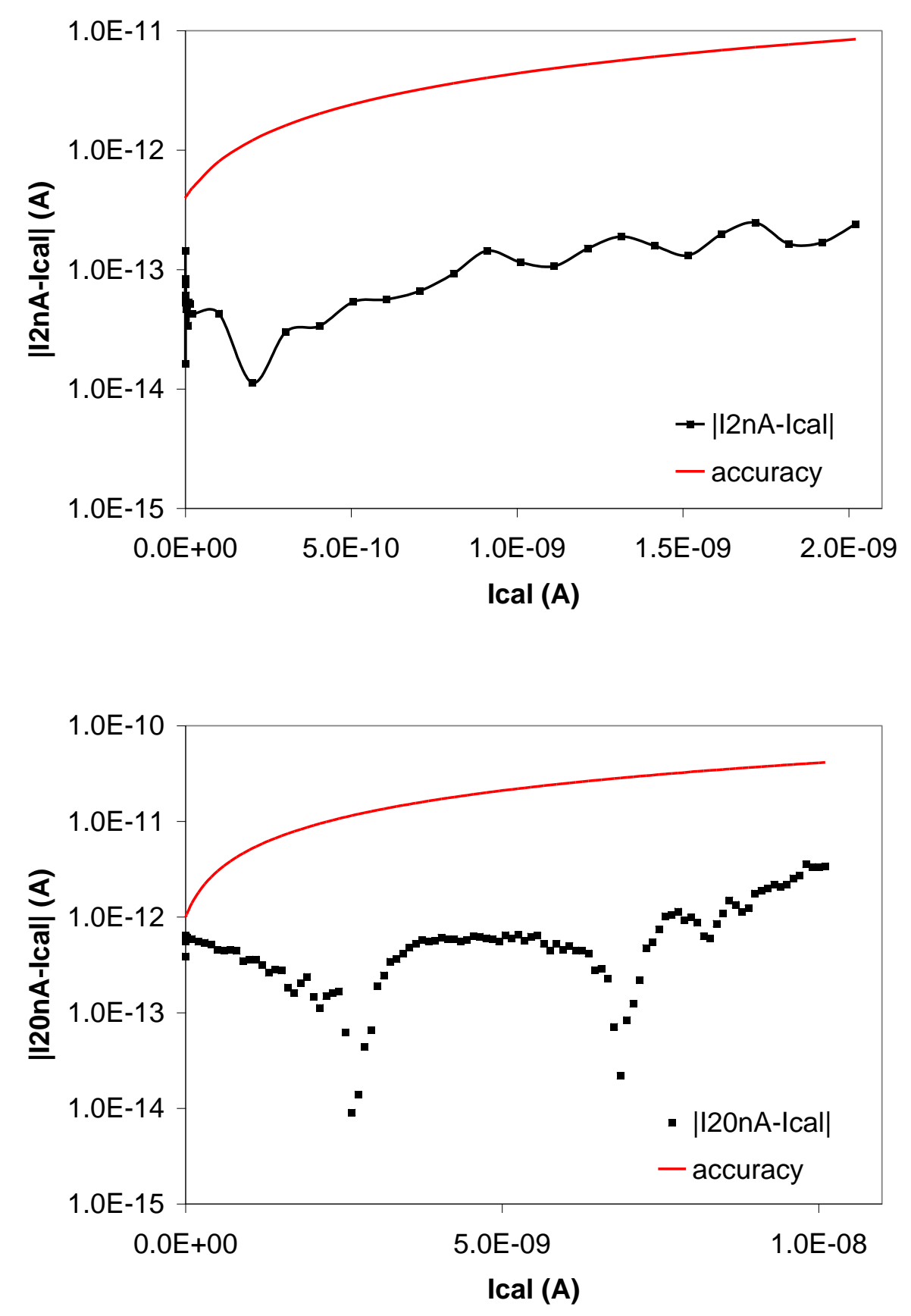

Figure 6. $|I-I c a l|$ and the accuracy (top: $2 \mathrm{nA}$ range and bottom: $20 \mathrm{nA}$ range).

\section{CONCLUSION}

We present a method based on Ohm's Law to calibrate the Keithley 6485 picoammeter with the calibration error less than 0.4 pico-ampere $(\mathrm{pA})$, which is specified as the highest sensitivity of this instrument. The calibration results show that the difference between the measurement current and the calibration current are within the accuracy specification. The maximum calibration difference between measurement current and the calibration current in $2 \mathrm{nA}$ range is far less than the highest accuracy specified. 


\section{ACKNOWLEDGMENTS}

This work is supported by Southern Methodist University and the Hamilton Scholarship. The authors would like to thank Dr. Datao Gong for his help in constructive discussions and technical help in fitting some of the curves using ROOT [5].

\section{REFERENCES}

1. Agilent 34401A Multimeter Data Sheet, Agilent Technologies, Inc., Santa Clara, CA, 2007.

2. Model $24101100 \mathrm{~V}$ SourceMeter Service Manual, 2nd ed., Keithley Instruments, Inc., Cleveland, OH, 1998.
3. Model 6485 Picoammeter User's Manual, 1st ed., Keithley Instruments, Inc., Cleveland, OH, 2003.

4. RX-1M Hi-Meg Datasheet, Ohmite Manufacturing Co., Rolling Meadows, IL, 2006.

5. ROOT - An Object Oriented Data Analysis Framework, Rene Brun and Fons Rademakers, Proceedings AlHENP'96 Workshop, Lausanne, Sep. 1996, Nucl. Inst. \& Meth. in Phys. Res. A 389 (1997) 81-86. See also http://root.cern.ch/.

\section{www.uni.edu/ajur}

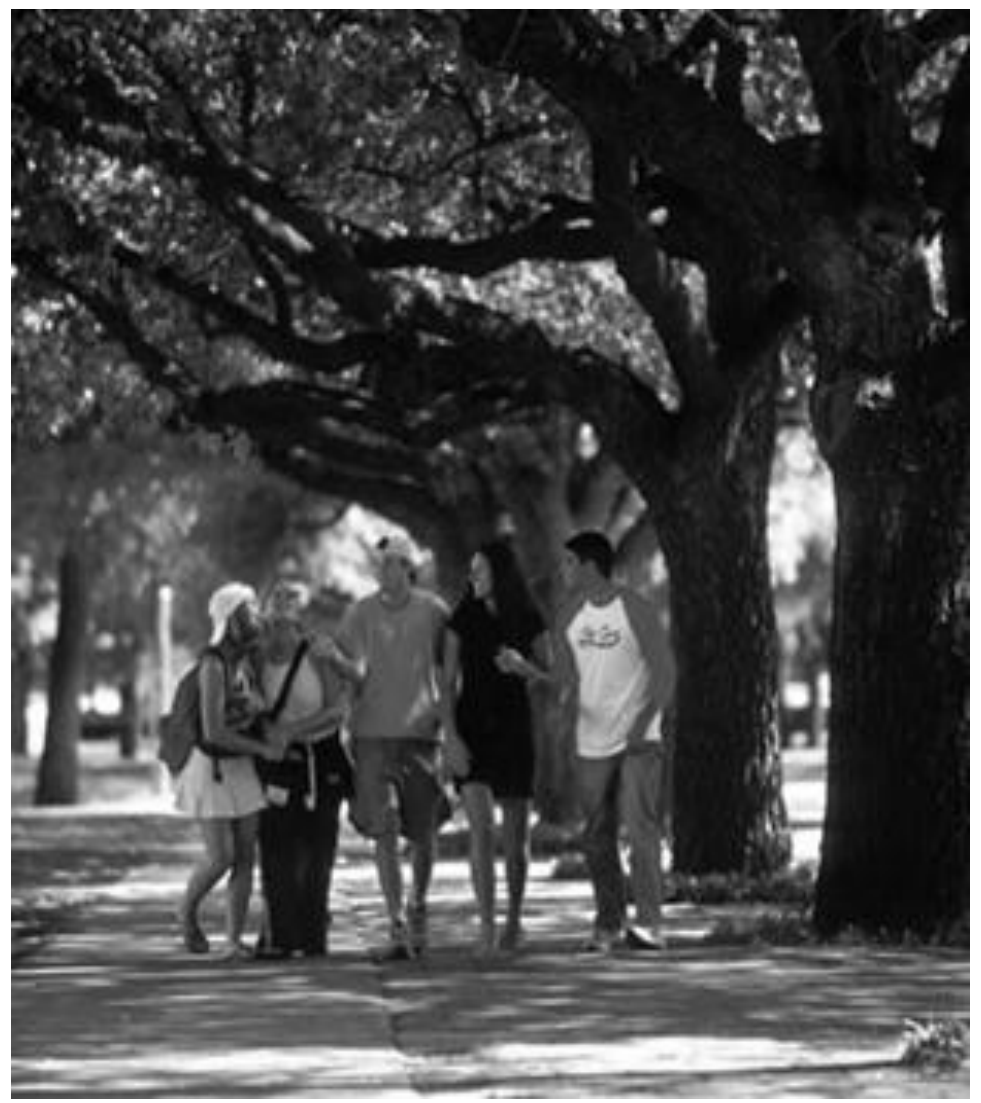

A century of molding scholars into leaders

At SMU, we believe that scholars are taught, and leaders are mentored. We have a 100-year history of graduating scholars who know how to lead, and our recent ranking among the nation's best colleges shows that with drive and experience, our graduates can count on becoming prized assets in their professions. Our class sizes are small, with an average faculty-to-student ratio of 12 to 1 . And our undergraduate professors are world-renowned scholars and researchers who will get to know you and take a personal interest in your success. With more than 100 majors and minors, as well as accelerated $4+$ 1 Master's programs in some schools, chances are SMU has an outstanding academic program that will open doors in your field that you may not have even known were there.

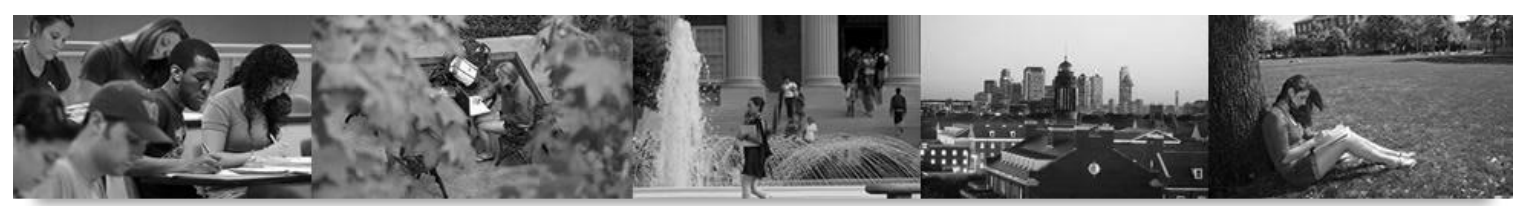

www.smu.edu/ 